\title{
Rapid Determination of 7-Hydroxycoumarin Using a Nanogold/ Poly-thionine Modified Glass Carbon Electrode
}

\author{
Yanjie Zheng, ${ }^{* 1, * 2, * 3 \dagger}$ Tianhua Zhong, ${ }^{* 4}$ Yichun XU, ${ }^{* 1}$ Li ChEn, ${ }^{* 1}$ Xinyang YIN, ${ }^{* 1}$ Fei LIN, ${ }^{* 1}$ \\ Qiang DAI, ${ }^{* 1}$ Shaohuang WENG, ${ }^{* 1, * 2 \dagger}$ and Xinhua LIN $* 1, * 2 \uparrow$
}

*1 Department of Pharmaceutical Analysis, School of Pharmacy, Fujian Medical University, No 1 Xueyuan Road, Minhou, Fuzhou 350122, China

*2 Nano Biomedical Technology Research Center, Fujian Medical University, No 1 Xueyuan Road, Minhou, Fuzhou 350122, China

*3 Fujian Key Laboratory of Drug Target Discovery and Structural and Functional Research, Fujian Medical University, No 1 Xueyuan Road, Minhou, Fuzhou 350122, China

*4 Key Laboratory of Marine Biogenetic Resources, Third Institute of Oceanograpy, Ministry of Natural Resources, No 178 University Road, Xiamen 361005, China

\begin{abstract}
This paper presents a novel voltametric procedure for 7-hydroxycoumarin determination by using a nanogold/polythionine modified electrode. The characterization of nanomaterials has been conducted by scanning electron microscopy (SEM) and electrochemical methods. The electrochemical sensing of 7-hydroxycoumarin was investigated by cyclic voltammetry $(\mathrm{CV})$ and differential pulse voltammetry (DPV). By combining the excellent electrocatalytic property of nanogold and polymer materials, this sensor shows an improved electrochemical response for 7-hydroxycoumarin detection with a good linear relationship in the range of $5.0 \times 10^{-6}-3.0 \times 10^{-5} \mathrm{~mol} \mathrm{~L}^{-1}$; the detection limit was $1.0 \times 10^{-6}$ mol L ${ }^{-1}$. This method solves the problem that 7-hydroxycoumarin cannot be accurately quantified on a bare glassy carbon electrode, and also improves the detection sensitivity. This is expected to play a huge potential in the quantitative analysis of quality control, plasma concentration monitoring and mechanism research in vivo of this drug.
\end{abstract}

Keywords 7-Hydroxycoumarin determination, nanogold, poly-thionine, cyclic voltammetry, differential pulse voltammetry

(Received September 16, 2020; Accepted November 10, 2020; Advance Publication Released Online by J-STAGE November 20, 2020)

\section{Introduction}

Coumarin is a kind of compound with a benzo- $\alpha$-pyrone nucleus, which is a natural polyphenolic phytochemical widely distributed in the plant kingdom. ${ }^{1,2}$ Coumarin compounds have attracted great attention because of their biological activity with medicinal value, such as being anti-inflammatory, ${ }^{3}$ anticoagulant, ${ }^{4}$ antibacterial ${ }^{5}$ and antiviral, ${ }^{6,7}$ while also owning anti-cancer activities. ${ }^{8,9}$ 7-Hydroxycoumarin (structural formula as shown in Fig. 1) is a derivative of coumarin. Based on an in-depth study of its biological activity, increasing potential applications have been found. For instance, Anegundi and

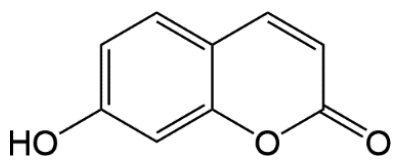

Fig. 1 Structure of 7-hydroxycoumarin.

† To whom correspondence should be addressed.

E-mail: gillzheng@mail.fjmu.edu.cn (Y. Z.); wengshaohuang@ 163.com (S. W.); xh11963@sina.com (X. L.)
Pancharatna used zebrafish embryo angiogenesis as a biological model, and proved an anti-angiogenic effect of 7-hydroxycoumarin, which was expected to play a role as a tumor angiogenesis inhibitor in the early treatment of cancer. ${ }^{10}$ $\mathrm{Li}$ et al. reported that 7-hydroxycoumarin was promising to be an antidote to MG while preventing the progression of MGrelated diseases for protecting $\mathrm{HepG} 2$ cells from methyl glyoxal (MG)-induced toxicity through the NRF2 activation pathway. ${ }^{11}$ Forthermore, Ramasamy et al. demonstrated that 7-hydroxycoumarin could lower ultraviolet B-induced oxidation and $\mathrm{NF}-\kappa \mathrm{B}$ activation, while also regulating the expression of inflammatory/photoaging molecules in human dermal fibroblast cells. The photoprotective properties indicate its potential as a sunscreen ingredient to protect skin from exogenous and endogenous harmful substances. ${ }^{12}$ In view of the many medicinal values of 7-hydroxycoumarin, the establishment of a rapid, sensitive and accurate detection method for its quantification is of certain significance for an intensive study concerning a rational use of this drug and its pharmacokinetics effect in vivo.

Until now, the methods for 7-hydroxycoumarin detection mainly focus on high-performance liquid chromatography, ${ }^{13,14}$ capillary electrophoresis ${ }^{15,16}$ and thin-layer chromatographicfluorescence. ${ }^{17}$ The coupling of these techniques may provide high selectivity of the assay, but also bring some disadvantages of operating complexity, time and reagent consuming and high 


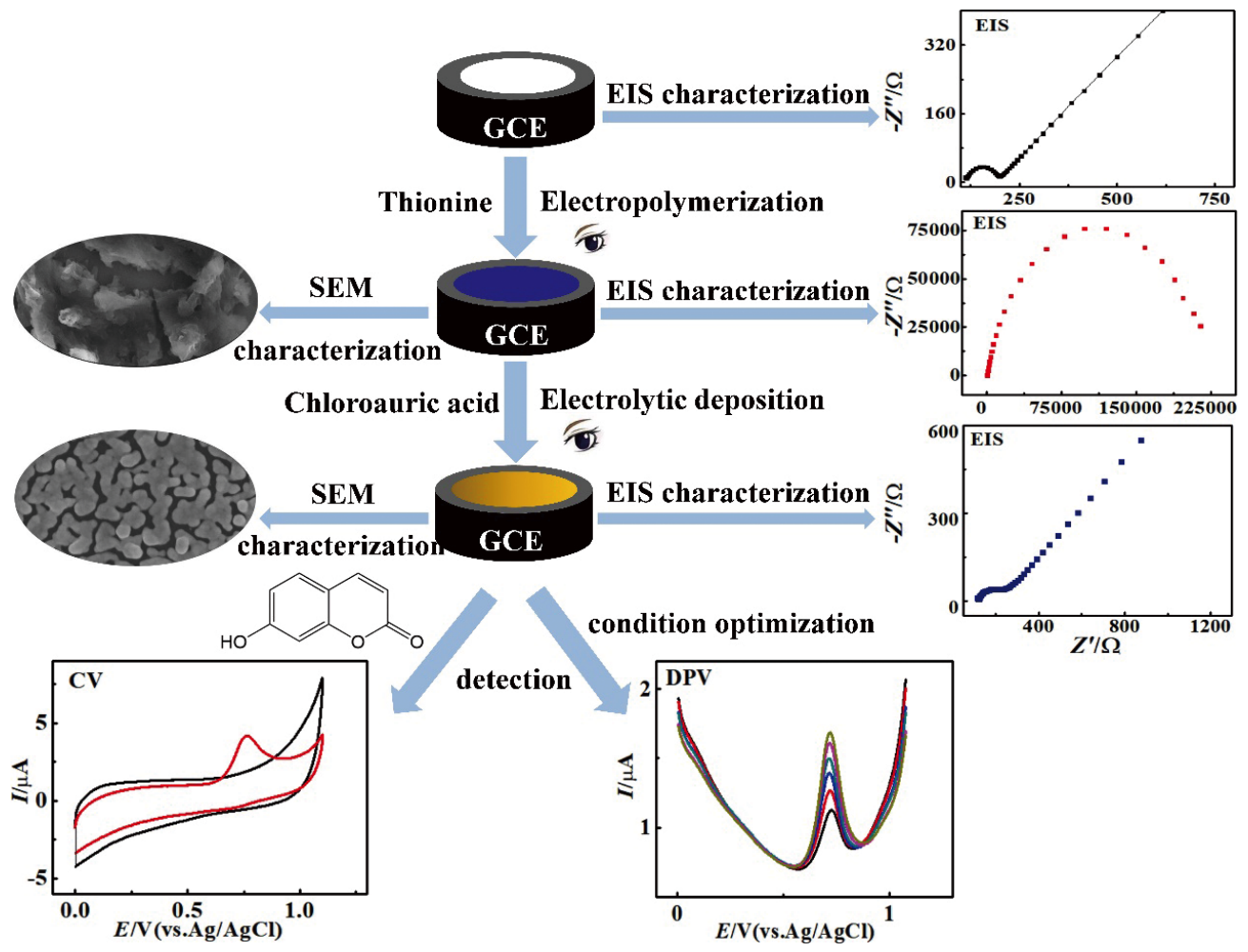

Fig. 2 Schematic diagram of the determination of 7-hydroxycoumarin.

cost. Therefore, it is essential to develop simple, rapid methods for their determination in routine analysis. Among various methods, electrochemical methods have been widely used for the detection of drugs and biological samples due to their advantages of easy operation, rapid response, low cost and being less time consuming. ${ }^{18-21}$ On account of the weak electroactive and irreversible property of 7-hydroxycoumarin, it is difficult to be determined accurately on a bare electrode directly. Wang and colleagues have found that a linear function of 7-hydroxycoumarin concentration was in the range from 0.5 to 5 mM. ${ }^{22}$ Thus, how to improve the electrochemical response of 7-hydroxycoumarin becomes the key problem to be solved in designing an electrochemical method. Conductive polymers have generally been used as ideal chemically modified materials for electrodes due to their good stability, excellent electrical conductivity, and strong electrocatalytic activity. ${ }^{23-26}$ Consequently, how to improve the detection sensitivity so as to achieve the detection of 7-hydroxycoumarin by electrochemical means could be a problem worth considering. Thionine, as a redox dye, ${ }^{27,28}$ could be fixed on the electrode surface by polymerization, while this conductive film could be a redox mediator with good redox reversibility, stability and a fast charge-transfer capacity. ${ }^{29-31}$ Moreover, as one of the inert metal nanoparticles, nanogold is widely used for electrode interface modification due to their high surface-to-volume ratio and surface activity, excellent electrocatalytic property and desirable biocompatibility. ${ }^{32-34}$ On account of the good conductivity of nanogold, it can improve electron transfer and amplify electrochemical signals of electroactive molecules. ${ }^{35}$ When polymer film combined with nanogold materials was modified on the electrode surface successively, the improved properties of the composites could be beneficial for molecular determination with low electrochemical activity. ${ }^{36}$

In this paper, nanogold/poly-thionine composite modified electrodes were prepared by electropolymerization and electrodeposition, respectively. The surface morphology and electrochemical properties of the modified electrodes were studied using scanning electron microscopy (SEM) and electrochemical methods, and the electrochemical behavior of 7-hydroxycoumarin on the modified electrode was investigated. Owing to the remarkable electrocatalytic effect of a nanogold/ poly-thionine composite on 7-hydroxycoumarin, the modified electrode was used as a working electrode to determine the content of 7-hydroxycoumarin successfully; the mechanism of 7-hydroxycoumarin determination by this method is shown in Fig. 2. It provides a new idea for quality control of the drug and a good electrochemical sensing platform for drug monitoring in complex biosamples that is simple, rapid, stable and green.

\section{Experimental}

\section{Reagents and chemicals}

Thionine was obtained from Sigma-Aldrich (St. Louis, MO, USA). Chloroauric acid was purchased from Sinopharm Chemical Reagent Co., Ltd. (China). 7-Hydroxycoumarin was from Beijing Bailingwei Technology Co., Ltd. (China). All other chemicals were of analytical grade and used without any further purification. Phosphate buffer solutions (PBS) were prepared by mixing stock solutions of $10 \mathrm{mM} \mathrm{NaCl}$ and $10 \mathrm{mM}$ $\mathrm{NaH}_{2} \mathrm{PO}_{4}-\mathrm{Na}_{2} \mathrm{HPO}_{4}$, and then adjusting the $\mathrm{pH}$ with a $50 \mathrm{mM}$ $\mathrm{H}_{3} \mathrm{PO}_{4}$ or $50 \mathrm{mM} \mathrm{NaOH}$. $\left[\mathrm{Fe}(\mathrm{CN})_{6}\right]^{3-14}$ solution acting as an electrolyte solution in electrochemical impedance spectrum (EIS) analysis containing $10 \mathrm{mM} \mathrm{K} \mathrm{K}_{3}\left[\mathrm{Fe}(\mathrm{CN})_{6}\right]-\mathrm{K}_{4}\left[\mathrm{Fe}(\mathrm{CN})_{6}\right]$, $0.1 \mathrm{M} \mathrm{KCl}$. An acetate buffer solution (ABS) was prepared by $0.2 \mathrm{M} \mathrm{HAc}$ and $\mathrm{NaAc}$, and pH was adjusted to 4.5 with $0.2 \mathrm{M}$ HAc. All solutions were prepared with double-distilled water. 

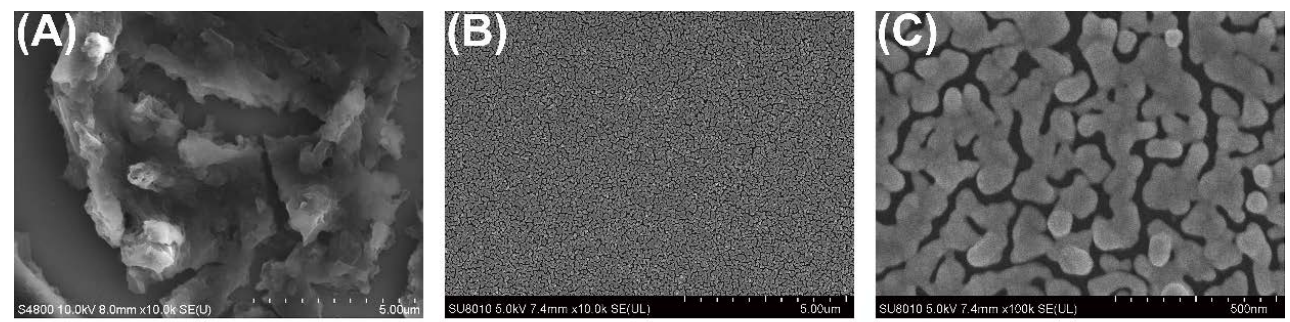

(D)

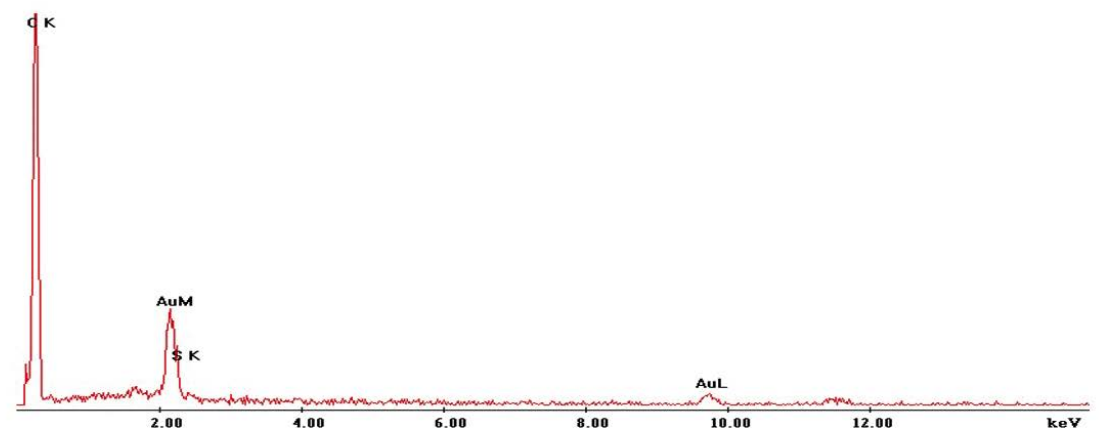

Fig. 3 SEM images of poly-thionine (A) and a nanogold/poly-thionine modified electrode (B, C) with different magnification (A and $\mathrm{B}, \times 10.0 \mathrm{k} ; \mathrm{C}, \times 100 \mathrm{k}$ ) and EDS (D) results of the surface of polythionine modified electrode.

\section{Apparatus}

The morphology of nanogold/poly-thionine was analyzed by using a SU8010 Ultra-high Resolution Scanning Electron Microscope (Hitachi, Japan). Electrochemical impedance spectroscopy were performed by employing an Autolab electrochemical workstation (PGSTAT 302F, Netherlands). All electrochemical measurements were carried out on a CHI 660D electrochemical workstation (Shanghai $\mathrm{CH}$ Instrument, China). A traditional three-electrode system was used for all electrochemical experiments, and the working electrode was a bare or modified glassy carbon electrode. A sliver-sliver chloride electrode $(\mathrm{Ag} / \mathrm{AgCl})$ and a platinum electrode were used as the reference and the auxiliary electrode, respectively.

\section{Preparation of poly-thionine modified electrode}

A bare glassy carbon electrode (GCE, $\Phi=3 \mathrm{~mm}$ ) was polished successively with $0.3,0.05 \mu \mathrm{m}$ of $\mathrm{Al}_{2} \mathrm{O}_{3}$ slurry. Then, it was rinsed with double-distilled water and sonicated in a $\mathrm{HNO}_{3}$ solution $\left(\mathrm{HNO}_{3}: \mathrm{H}_{2} \mathrm{O}=1: 1\right)$, and then ethanol and doubledistilled water in turn for $10 \mathrm{~min}$. After being cleaned, an electrochemical modification of GCE was performed using cyclic voltammetry $(\mathrm{CV})$ in a HAc-NaAc buffer ( $\mathrm{pH} 4.5$ ) containing $1.0 \mathrm{mmol} \mathrm{L}^{-1}$ thionine solution in the potential range of $-0.4-1.5 \mathrm{~V}$ for 25 cycles at a sweep rate of $100 \mathrm{mV} \mathrm{s}^{-1}$. $^{37}$ After electropolymerization, the electrode was thoroughly rinsed with distilled water for further use.

Preparation of a nanogold/poly-thionine modified electrode

Electrodeposition was used to prepare a nanogold/polythionine modified electrode; the polymer electrode was placed in a $0.1 \mathrm{mmol} \mathrm{L}^{-1}$ sulfuric acid solution containing $5.0 \mathrm{mmol} \mathrm{L}^{-1}$ of chloroauric acid, and electrodeposition was enforced under $\mathrm{CV}$ sweeping over $-1.5-0 \mathrm{~V}$ at $100 \mathrm{mV} \mathrm{s}^{-1}$ for 5 cycles. After deposition, the modified electrode was rinsed thoroughly with distilled water.
Table 1 EDAX ZAF quantification

\begin{tabular}{ccc}
\hline Element & Weight, $\%$ & Atom, $\%$ \\
\hline CK & 23.24 & 82.11 \\
SK & 1.22 & 1.62 \\
AuL & 75.54 & 16.28 \\
Total & 100.00 & 100.00 \\
\hline
\end{tabular}

\section{Results and Discussion}

SEM characterization of poly-thionine and nanogold/polythionine modified electrodes

A SEM image provides detailed information about the surface morphology of a modified electrode. As shown in Fig. 3A, a slight loose, fog-like, heterogeneous film appeared on the electrode surface which indicated that thionine successfully polymerized on the surface of GCE. On the basis of the polythionine modified electrode, when electrodeposition was conducted using chloroauric acid (Fig. 3B), the interface of GCE was covered with a relatively uniform nanogold with a certain thickness in a low magnification field of view $(\times 10.0 \mathrm{k})$; when observing in $\times 100 \mathrm{k}$ (Fig. 3C), a three-dimensional structure could be clearly seen to be different from that on the bare glass carbon substrate electrode, further indicating that a nanogold film indeed partially covered on the electrode. Furthermore, the elemental analysis was investigated with EDX, as shown in Fig. 3D. EDS analysis obtained from the surface of a nanogold/poly(thionine)-modified electrode confirmed the stable and main ingredients of $\mathrm{Au}$ and $\mathrm{C}$ elements along with a small amount of $\mathrm{S}$ (Table 1) confirming the chemical state of the nanogold/poly(thionine) combined with the SEM image.

Electrochemical characterization of a nanogold/poly-thionine modified electrode

Electrochemical impedance spectroscopy (EIS) can give information on impedance changes of the electrode surface in 

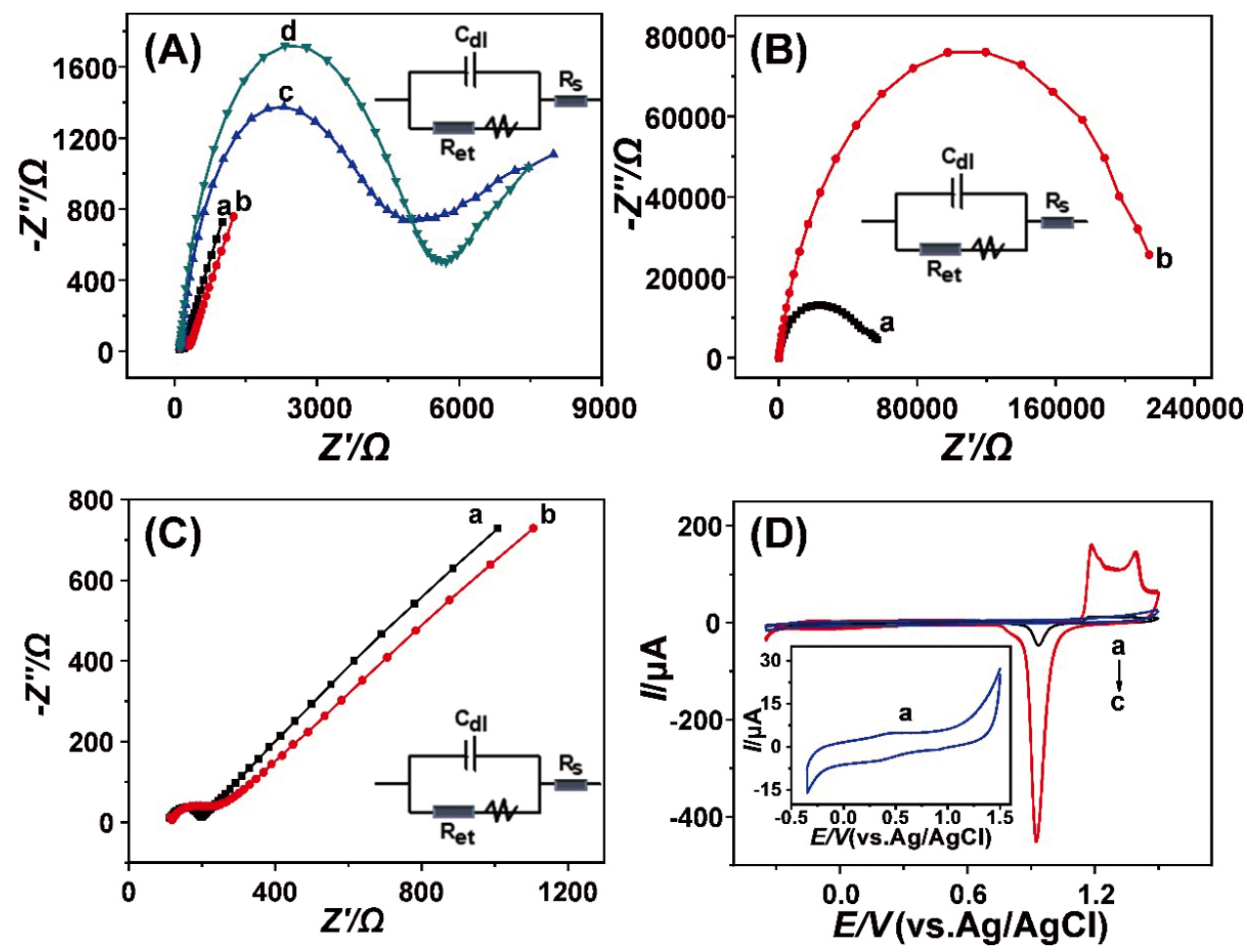

Fig. 4 Electrochemical impedance spectrum (EIS) of different electrodes. (A) Bare electrode (a); poly-thionine modified electrode with 1 cycle (b); 2 cycles (c); 3 cycles (d). (B) 5 Cycles (a); 25 cycles (b). (C) Bare electrode (a); nanogold/poly-thionine modified electrode (b). (D) CV curves of bare GCE (a), bare gold electrode (b) and nanogold/poly-thionine modified electrode (c) in $0.5 \mathrm{M} \mathrm{H}_{2} \mathrm{SO}_{4}$ solution at a scan rate of $100 \mathrm{mV} / \mathrm{s}$.

the modification process. EIS was thus also used to evaluate the electron transfer efficiency at the different stages of interfacial electrode shown in Fig. 4. With an increase of the polymerization cycle, the electrontransfer resistance $\left(R_{\mathrm{et}}\right)$ increased significantly with increasing polymerization cycles (Fig. 4A, curve b-1, c-2, d-3; Fig 4B, curve a-5, b-25 cycles, respectively) when aggregated for 25 cycles (Fig. 4B, curve b), the $R_{\text {et }}$ value was nearly $210000 \Omega$. It was evident that a poly-thionine film on a bare GCE surface was already formed after one cyclic of electropolymerization. Subsequently, when deposited by chloroauric acid on the poly-thionine modified electrode (Fig. 4C, curve b), $R_{\text {et }}$ decreased sharply and nearly close to the response of a bare GCE interface (Fig. 4C, curve a), indicating that the deposition behavior of gold nanoparticles on the film layer was conducive to the electron exchange for $\left[\mathrm{Fe}(\mathrm{CN})_{6}\right]^{3-14-}$ on the electrode surface. By combining the diagrams of SEM and EIS, it could be determined that nanogold film had formed on the poly-thionine modified electrode successfully, which could play a vital role in accelerating electron transfer. Furthermore, the electrochemical characterization of nanogold/ poly-thionine modified GCE electrode was performed in $0.5 \mathrm{~mol} \mathrm{~L}^{-1} \mathrm{H}_{2} \mathrm{SO}_{4}$ at a scan rate of $100 \mathrm{mV} \mathrm{L}^{-1}$. For a comparison, the voltammetric behaviors of a bare glass carbon electrode and a bare gold electrode with the same geometric surface area were jointly investigated by using cyclic voltammetry. As shown in Fig. 4D, there was no obvious response on the bare glassy carbon electrode in a sulfuric acid solution (Fig. 4D, curve a), while the current signal was significantly enhanced on the nanogold/poly-thionine modified electrode (Fig. 4D, curve c) than that of the bare gold electrode (Fig. 4D, curve b). By assuming the reduction of a monolayer

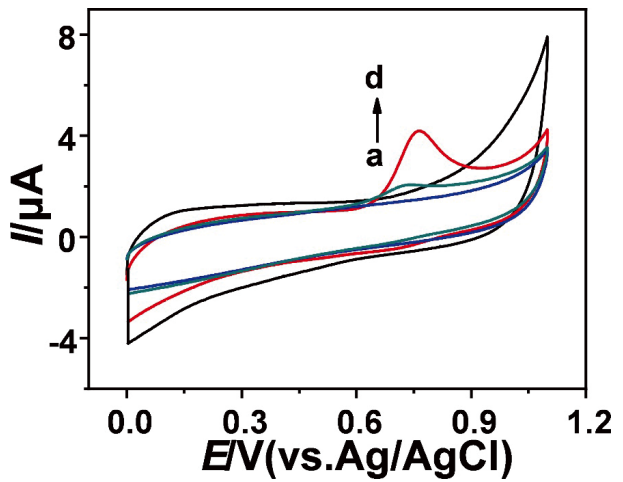

Fig. $5 \mathrm{CV}$ curves of $15.0 \mu \mathrm{mol} \mathrm{L}-1$ 7-hydroxycoumarin at bare electrode and $5.0 \mu \mathrm{mol} \mathrm{L}-1$ 7-hydroxycoumarin at nanogold/polythionine modified electrode in PBS of $\mathrm{pH} 7.4$ (scan rate: $100 \mathrm{mV} \mathrm{s}^{-1}$ ).

of gold oxide requires $386 \mu \mathrm{C} \mathrm{cm}^{-2},{ }^{38}$ the real surface of the nanogold/poly-thionine) modified electrode was approximately 9.7-times larger than that of a bare gold electrode with the same geometric area. Upon combining the graphs of Figs. 3 and 4, obviously, nanogold had successfully been deposited on the surface of the pretreated electrode by thionine, resulting in the electrode having a larger electroactive surface and a higher conductivity.

\section{Electrochemical behavior of 7-hydroxycoumarin}

Figure 5 shows CV curves of bare and nanogold/polythionine modified GCE in a PBS solution with and without 


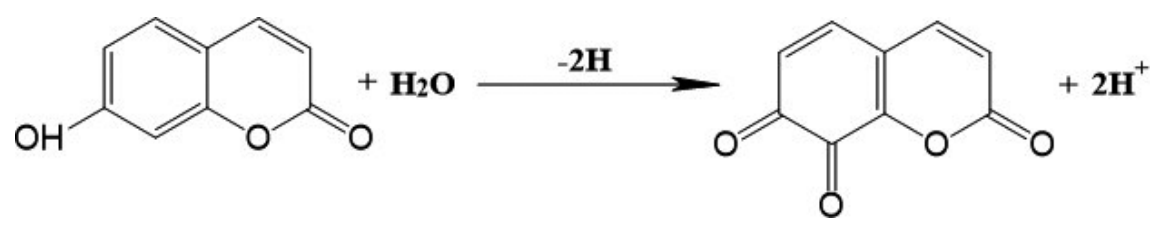

Fig. 6 Electrochemical behavior of 7-hydroxycoumarin on a nanogold/poly-thionine modified electrode.

7-hydroxycoumarin. In a PBS solution, there was no obvious current response signal on either bare GCE (Fig. 5, curve a) or a nanogold/poly-thionine modified electrode (Fig. 5, curve b); when adding $5.0 \mu \mathrm{mol} \mathrm{L} \mathrm{L}^{-1}$ 7-hydroxycoumarin, no obvious peak current response could be observed on GCE, basically coinciding with the response of the PBS solution. Even though the

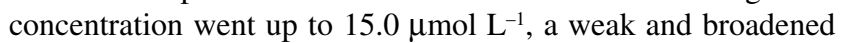
oxidation peak appeared at $0.712 \mathrm{~V}$ (Fig. 5, curve c) which could not be accurately quantified. However, a well-shaped oxidation peak appeared at $0.764 \mathrm{~V}$ after adding $5.0 \mu \mathrm{mol} \mathrm{\textrm {L } ^ { - 1 }}$ 7-hydroxycoumarin on the nanogold/poly-thionine modified electrode (Fig. 5, curve d). The oxidation peak potential was positively shifted by $52 \mathrm{mV}$ with a significantly improved peak shape and an increased peak current in contrast to that at bare GCE. The remarkable peak current enhancement can undoubtedly be attributed to the unique structure and properties of nanogold/poly-thionine (such as large specific area, strong adsorptive ability, and subtle electronic properties). ${ }^{39-42}$ In short, this new approach could be suitable for the direct determination of 7-hydroxycoumarin and improve the detection sensitivity concurrently. The possible electrochemical reaction of 7-hydroxycoumarin on the modified electrode is illustrated in Fig. 6.

Furthermore, in order to make sure the different electrochemical response of 7-hydroxycoumarin on each modified electrode interface, the electrocatalytic properties of different modified electrodes (such as poly-thionine, nanogold and nanogold/polythionine modified electrode) were used as the working electrodes for 7-hydroxycoumarin detection and the electrocatalytic properties were compared. As shown in Fig. 7, a weak oxidation peak with an unsatisfactory peak shape and peak height appeared on the bare (curve a), poly-thionine (curve b) and nanogold modified GCE (curve c) at the same concentration at 0.712, 0.796 and $0.801 \mathrm{~V}$, respectively. However, the peak current response at a nanogold/poly-thionine modified electrode increased significantly (curve d) with a distinctly improved shape of the peak. This shows that the electrode modified by the nanogold/poly-thionine composite material has a better and more significant electrocatalytic effect on 7-hydroxycoumarin than other monolayer film ones, which makes the quantitative determination of 7-hydroxycoumarin possible.

\section{Optimization of experimental conditions}

The number of deposition cycles directly determines the thickness of the polymer film, and a proper increase in the thickness of the nanogold also the influnces electrostatic interaction between the modified electrode and 7-hydroxycoumarin. Thus, the influence of different deposition behaviors on oxidation peak was investigated. As shown in Fig. S1, when the number of electrodeposition cycles was 5 , the peak appeared at $0.764 \mathrm{~V}$ (Fig. S1, curve b) with the peak current being reached at $3.039 \mu \mathrm{A}$. As cycle number increased to 10 , the peak potential moved to $0.788 \mathrm{~V}$ (curve c) with the peak current decreasing to $1.882 \mu \mathrm{A}$. Combined with the $\mathrm{CV}$ curves in Fig. 5, the increasing deposition amount of nanagold increased

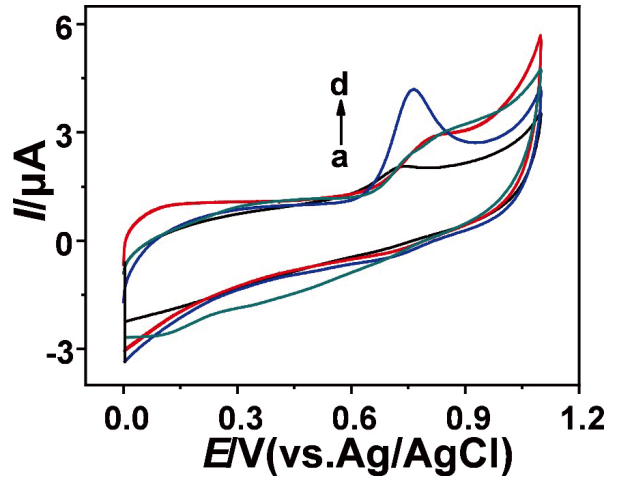

Fig. $7 \mathrm{CV}$ curves of $5.0 \mu \mathrm{mol} \mathrm{L} \mathrm{L}^{-1}$ 7-hydroxycoumarin at a bare electrode (a), poly-thionine modified electrode (b), nanogold modified electrode (c) and nanogold/poly-thionine electrode (d) in PBS (pH 7.4) (scan rate: $100 \mathrm{mV} \mathrm{s}^{-1}$ ).

the coverage on the surface of poly-thionine film, leading to the reduction capacity of electron transfer between the active component and the 3D electrode interface to a certain extent, and then, the current signal decreased. Therefore, it is advisable to choose 5 cycles for the preparation of nanogold film.

The electrochemical oxidation behaviors of 7-hydroxycoumarin in various media, such as PBS, Britton-Robinson buffer, HAc$\mathrm{NaAc}$ buffer, Tris- $\mathrm{HCl}$ buffer and $\mathrm{NH}_{3} \cdot \mathrm{H}_{2} \mathrm{O}-\mathrm{NH}_{4} \mathrm{Cl}$ buffer, were compared. The best oxidation response was obtained in $\mathrm{pH} 7.4$ PBS since the peak current is the highest and the peak shape was well-defined. In addition, the influence of the $\mathrm{pH}$ value on the oxidation peak current of 7-hydroxycoumarin was also examined in a PBS solution (Fig. S2). The $E_{\mathrm{pa}}$-versus- $\mathrm{pH}$ graph clearly indicates that the catalytic peak shifts to a more negative potential with an increase of the $\mathrm{pH}$. The oxidation peak potential has a good linear relationship with the $\mathrm{pH}$ value and the linear equation was $E_{\mathrm{pa}}(\mathrm{V})=1.1574-0.04914 \mathrm{pH}$ in the range of $\mathrm{pH} 4.0$ - 10.0, with a correlation coefficient of 0.9952 . The peak current increases as the $\mathrm{pH}$ increases and reaches the largest at $\mathrm{pH} 7.4$, and subsequently, the peak current signal decreases as the $\mathrm{pH}$ continues to increase. Therefore, a PBS of $\mathrm{pH} 7.4$ was chosen as the determining medium.

Furthermore, the effect of the scan rate on the anodic peak current of 7-hydroxycoumarin was studied in this paper. As the scan rate increased, $I_{\mathrm{pa}}$ was directly proportional to the scan rate $(v)$ over the range of $40-160 \mathrm{mV} \mathrm{s}^{-1}$ (as shown in Fig. S3). The linear-regression equation was $I_{\mathrm{pa}}=0.0063 v+0.8161$ with a correlation coefficient of 0.9958 , suggesting a surfacecontrolled process on the modified electrode surface. The excessive sweep speed would lead to an increase of charging current and a decrease of the peak current, so $100 \mathrm{mV} \mathrm{s}^{-1}$ was chosen as the optimized condition.

\section{Electrochemical detection of 7-hydroxycoumarin}

Since DPV has a much higher current sensitivity and better 
resolution than $\mathrm{CV}$, it was used for determining the 7-hydroxycoumarin concentration at the nanogold/polythionine modified electrode and estimating the lower limit of detection. As shown in Fig. 8, the dependence of the peak current on the concentration of 7-hydroxycoumarin was in a linear relationship in the range of 5.0 to $30.0 \mu \mathrm{mol} \mathrm{L}^{-1}$. The linear equation is $I_{\mathrm{pa}}=0.0217 C+0.2739$ with a correlation coefficient of 0.9951 , and the detection limit is $1.010^{-6} \mathrm{~mol} \mathrm{~L}^{-1}$ $(S / N=3)$. The relative standard deviation of the same electrode in 9 successive scans is $2.1 \%$ for $15.0 \mu \mathrm{mol} \mathrm{L}^{-1}$ 7-hydroxycoumarin and $3.2 \%$ for interelectrodes, indicating that this modified electrode has an excellent reproducibility. This proposed method has effectively improved the detection sensitivity of this drug, which is difficult to be accurately quantified on a bare GCE. The analytical performance of several strategies containing HPLC and CE along with DPV approaches is summarized and compared in Table 2. The LOD of this proposed assay is even lower than those of previously reported approaches. ${ }^{13-16,22}$ Although this method could not achieve a higher sensitivity than a reported, ${ }^{17}$ this method makes 7-hydroxycoumarin detection by the electrochemical method more sensitive than that in previously literature, which has never been deeply considered after Wang reported. ${ }^{22}$ Meanwhile, it is worth noting that this detection could meet the requirement of a rapid and accurate determination for 7-hydroxycoumarin with simple and facile operation, indicating its potential for the possible analysis of some drugs with weak electrochemical activity.

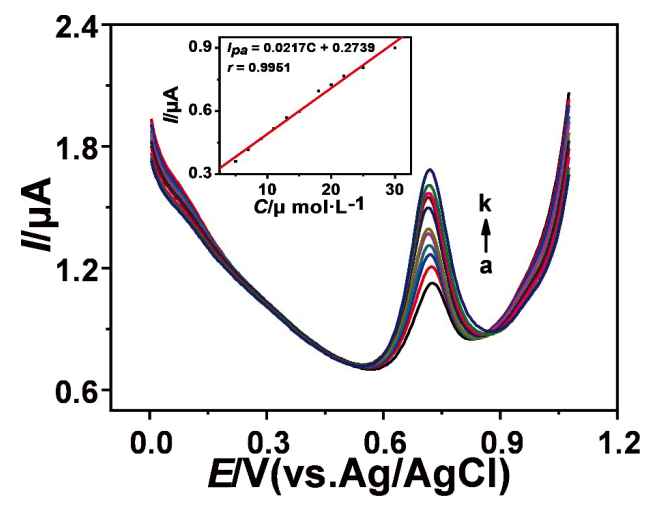

Fig. 8 DPV curves with varying amounts of 7-hydroxycoumarin on nanogold/poly-thionine modified electrode in PBS ( $\mathrm{pH} 7.4$ ) (from bottom to top: $5.0,7.0,9.0,11.0,13.0,15.0,18.0,20.0,22.0,25.0$, $30.0 \mu \mathrm{M})$. The inset shows a linear fitting curve between $I_{\mathrm{pa}}$ and the concentrations of 7-hydroxycoumarin.

\section{Interferences}

To evaluate the interferences of foreign species on the determination of 7-hydroxycoumarin, the effect of the presence of possible co-existence interference was investigated by DPV. In the study, no obvious interference was found concerning for the detection of $5.0 \mu \mathrm{mol} \mathrm{L}^{-1}$ 7-hydroxycoumarin when adding $100 \mathrm{M}$ of $\mathrm{K}^{+}, \mathrm{Zn}^{2+}, \mathrm{Ca}^{2+}, \mathrm{NO}^{3-}, \mathrm{Cl}^{-}, \mathrm{SO}_{4}{ }^{2-}$, sodium citrate, lactose into the mixture solution. The results indicate that the modified electrode possesses an excellent anti-interference capability for the determination of 7-hydroxycoumarin in a complex sample.

\section{Analytical application to human urine}

In order to evaluate the feasibility of the method in practical applications, the detection of 7-hydroxycoumarin in human urine was carried out. Three treated urine samples were respectively spiked with 5.0 and $10.0 \mu \mathrm{mol} / \mathrm{L}$ of 7-hydroxycoumarin. Table 3 shows that the measured recovery of 7-hydroxycoumarin in urine was between 96.6 and $103.7 \%$ with an RSD of less than $5.2 \%$. Thus, the measured recovery demonstrated the reliability and accuracy of the proposed method for the detection of 7-hydroxycoumarin in human urine samples, which can be an attractive candidate for drug monitoring.

\section{Conclusions}

In this study, a nanogold/poly-thionine modified electrode was successfully prepared. The SEM measurement indicates that this composite film could be assembled layer-by-layer on the surface of an electrode. This composite film has good electrocatalytic activity toward the oxidation of 7-hydroxycoumarin and significantly improves the detection sensitivity, which could probably solve some components analysis with weak electrochemical activity. Furthermore, this method has the advantages of simple preparation, low cost and

Table 3 The recovery of the proposed method in 50\% human serum

\begin{tabular}{ccccc}
\hline $\begin{array}{c}\text { Sample } \\
\text { number }\end{array}$ & $\begin{array}{c}C_{\text {added }} / \\
\mu \mathrm{M}\end{array}$ & $\begin{array}{c}C_{\text {tested }} / \\
\mu \mathrm{M}\end{array}$ & $\begin{array}{c}\text { Recovery, } \\
\%\end{array}$ & $\begin{array}{c}\text { RSD, } \\
\%\end{array}$ \\
\hline 1 & 5.0 & 5.07 & 101.4 & 4.5 \\
2 & & 4.83 & 96.6 & 5.2 \\
3 & & 4.89 & 97.8 & 2.7 \\
4 & \multirow{2}{*}{10.0} & 9.88 & 98.8 & 2.9 \\
5 & & 10.37 & 103.7 & 3.7 \\
6 & & 9.75 & 97.5 & 3.1 \\
\hline
\end{tabular}

Table 2 Comparison of the reported methods for 7-hydroxycoumarin detection

\begin{tabular}{llll}
\hline \multicolumn{1}{c}{ Detection strategies } & \multicolumn{1}{c}{ Linear range } & Detection limit & Ref. \\
\hline High-performance liquid chromatography & $3.08-616 \mu \mathrm{M}(0.5-100 \mu \mathrm{g} / \mathrm{mL})$ & $3.08 \mu \mathrm{M}(0.5 \mu \mathrm{g} / \mathrm{mL})$ & 13 \\
High-performance liquid chromatography & $30.8-616 \mu \mathrm{M}(5-100 \mu \mathrm{g} / \mathrm{mL})$ & $30.8 \mathrm{nM}(50 \mathrm{ng} / \mathrm{mL})$ & 14 \\
Capillary electrophoresis & $0-308 \mu \mathrm{M}(0-50 \mu \mathrm{g} / \mathrm{mL})$ & $6.17 \mu \mathrm{M}(1 \mu \mathrm{g} / \mathrm{mL})$ & 15 \\
Capillary zone electrophoresis & $0-150 \mu \mathrm{M}(0-24.3 \mu \mathrm{g} / \mathrm{mL})$ & $6.17 \mu \mathrm{M}(1 \mu \mathrm{g} / \mathrm{mL})$ & 16 \\
Thin-layer chromatographic-fluorescence & $0.0308-61.6 \mu \mathrm{M}(5-10000 \mathrm{ng} / \mathrm{mL})$ & $6.17 \mathrm{nM}(1 \mathrm{ng} / \mathrm{mL})$ & 17 \\
Differential pulse voltammetry at bare GCE & $0.5-5 \mathrm{mM}(81-810 \mu \mathrm{g} / \mathrm{mL})$ & $10 \mu \mathrm{M}(1.62 \mu \mathrm{g} / \mathrm{mL})$ & 22 \\
Differential pulse voltammetry at nanogold/poly-thionine & $5-30 \mu \mathrm{M}(0.81-4.86 \mu \mathrm{g} / \mathrm{mL})$ & $1 \mu \mathrm{M}(0.162 \mu \mathrm{g} / \mathrm{mL})$ & $\mathrm{This}$ work \\
modified GCE & & & \\
\hline
\end{tabular}


good stability, which can provide a new idea for the quality control of 7-hydroxycoumarin and the monitoring of its clinical urine or blood concentration.

\section{Acknowledgements}

The authors gratefully acknowledge the financial support of the National Science Foundation of China (21705021 and 21775023), Open project (HY201703) and COMRA program (DY135-B2-08) funded by Key Laboratory of Biological Genetic Resources from Ministry of Natural Resources, Medical Innovation Project of Fujian Province of China (2016-CX-44), Joint Funds for the Innovation of Science and Technology, Fujian Province (2019Y9011 and 2017Y9121).

\section{Supporting Information}

This material is available free of charge on the Web at http:// www.jsac.or.jp/analsci/.

\section{References}

1. D. Cao, Z. Liu, P. Verwilst, S. Koo, P. Jangjili, J. S. Kim, and W. Lin, Chem. Rev., 2019, 119, 10403.

2. F. Y. Jia, F. F. Su, M. Ran, and B. Zhang, Spectrosc. Spect. Anal., 2018, 38, 320.

3. Q. Wang, Y. Chen, G. Li, Z. Liu, J. Ma, M. Liu, D. Li, J. Han, and B. Wang, Bioorg. Med. Chem., 2019, 27, 2112.

4. N. C. Bang, A. Z. Abyshev, and D. Y. Ivkin, Pharm. Chem. J., 2019, 53, 419.

5. D. Dastan, P. Salehi, A. Aliahmadi, A. R. Gohari, H Maroofi, and A. Ardalan, Nat. Prod. Res., 2016, 30, 2747.

6. S. Mishra, A. Pandey, and S. Manvati, Heliyon, 2020, 6, e03217.

7. M. Z. Hassan, H. Osman, M. A. Ali, and M. J. Ahsan, Eur. J. Med. Chem., 2016, 123, 236.

8. A. Thakur, R. Singla, and V. Jaitak, Eur. J. Med. Chem., 2015, 101, 476.

9. L. Huang, Z. L. Feng, Y. T. Wang, and L. G. Lin, Chin. J. Nat. Medicines, 2017, 15, 881.

10. N. Anegundi and K. Pancharatna, Eur. Sci. J., 2017, 13, 53.

11. D. Li, N. Wang, J. Zhang, S. Ma, Z. Zhao, and E. M. Ellis, Chem. Biol. Interact., 2017, 276, 203.

12. R. Karthikeyan, G. Kanimozhi, N. R. Prasad, B. Agilan, M. Ganesan, S. Mohana, and G. Srithar, J. Photochem. Photobiol., B, 2016, 161, 170.

13. D. A. Egan and R. O'Kennedy, J. Chromatogr. B, 1992 , 582, 137.

14. D. P. Bogan and R. O'Kennedy, J. Chromatogr. B, 1996, 686, 267.

15. D. P. Bogan, B. Deasy, R. O'Kennedy, M. R. Smyth, and U. Fuhr, J. Chromatogr. B, 1995, 663, 371.

16. C. F. Duffy and R. O'Kennedy, J. Pharm. Biomed. Anal.,
1998, $17,1279$.

17. S. Cholerton, M. E. Idle, A. Vas, F. J. Gonzalez, and J. R. Idle, J. Chromatogr. B, 1992, 575, 325.

18. A. Özcan, S. İlkbaş, and A. A. Özcan, Talanta, 2017, 165, 489.

19. W. Guo, F. Pi, H. Zhang, J. Sun, Y. Zhang, and X. Sun, Biosens. Bioelectron., 2017, 98, 299.

20. S. Jafari, M. Dehghani, N. Nasirizadeh, and M. Azimzadeh, J. Electroanal. Chem., 2018, 829, 27.

21. Y. Li, W. Ye, Y. Cui, B. Li, Y. Yang, and G. Qian, J. Mol. Struct., 2020, 1209, 127986.

22. E. Dempsey, C. O'Sullivan, M. R. Smyth, D. Egan, and J. Wang, J. Pharm. Biomed. Anal., 1993, 11, 443.

23. M. M. Rahman, Y. J. Kim, and J. J. Lee, Bull. Korean Chem. Soc., 2017, 38, 27.

24. W. Silva, M. E. Ghica, and C. M. A. Brett, Electrochim. Acta, 2019, 317, 766.

25. M. Ding, Y. Zhou, X. Liang, H. Zou, Z. Wang, M. Wang, and J. Ma, J. Electroanal. Chem., 2016, 763, 25.

26. R. Rajaram, S. Anandhakumar, and J. Mathiyarasu, J. Electroanal. Chem., 2015, 746, 75.

27. Z. Ghafary, R. Hallaj, A. Salimi, and K. Akhtari, ACS Omega, 2019, 4, 15323.

28. T. Shamspur, Z. Biniaz, A. Mostafavi, M. TorkzadehMahani, and M. Mohamadi, IEEE Sens. J., 2018, 18, 4861.

29. Y. Lai, Y. Deng, G. Yang, S. Li, C. Zhang, and X. Liu, J. Biomed. Nanotechnol., 2018, 14, 1688.

30. C. Liu, J. Huang, and L. Wang, Microchim. Acta, 2018, 185,414

31. Y. Dai, X. Li, L. Fan, X. Lu, and X. Kan, Biosens. Bioelectron., 2016, 86, 741.

32. W. Silva, M. E. Ghica, C. M. A. Brett, R. Ajayi, and E. I. Iwuoha, Talanta, 2019, 195, 604.

33. N. B. Messaoud, M. E. Ghica, C. Dridi, M. B. Ali, and C. M. A. Brett, Sens. Actuators, B, 2017, 253, 513.

34. Y. Wang, Y. Xiong, J. Qu, J. Qu, and S. Li, Sens. Actuators, $B$, 2016, 223, 501 .

35. Q. Zhu, J. Bao, D. Huo, M. Yang, C. Hou, J. Guo, M. Chen, H. Fa, X. Luo, and Y. Ma, Sens. Actuators, B, 2017, 238, 1316.

36. Y. Wang, L. Wang, H. Chen, X. Hu, and S. Ma, ACS Appl. Mater. Inter., 2016, 8, 18173.

37. J. M. Bauldreay and M. D. Archer, Electrochim. Acta, 1983, 28, 1515.

38. W. Gao, X. H. Xia, J. J. Xu, and H. Y. Chen, J. Phys. Chem. $C, \mathbf{2 0 0 7}, 111,12213$.

39. Z. Li, Y. Huang, L. Chen, X. L. Qin, Z. Huang, Y. P. Zhou, Y. Meng, J. Li, S. Y. Huang, Y. Liu, W. Wang, Q. J. Xie, and S. Z. Yao, Sens. Actuators, B, 2013, 181, 280.

40. M. Portaccio, D. D. Tuoro, F. Arduini, M. Lepore, D. G. Mita, N. Diano, L. Mita, and D. Moscone, Biosens. Bioelectron., 2010, 25, 2003.

41. H. Y. Liu, G. F. Wang, D. L. Chen, W. Zhang, and C. J. Li, Sens. Actuators, B, 2008, 128, 414.

42. A. J. S. Ahammad, N. C. D. Nath, S. Kim, Y. Kim, and J. Lee, Bull. Korean Chem. Soc., 2011, 32, 779. 